HRJ

V.2 n.10 (2021)

Recebido: 06/09/2020

Aceito: 20/02/2021

\title{
Paresia do IV nervo com sinal de Bielschowsky positivo: um relato de caso
}

\author{
Isabela Porto Silva Costa ${ }^{1}$ \\ Talita Trindade França ${ }^{2}$ \\ Ana Clara Guerreiro Araújo de Gouvêa ${ }^{3}$ \\ Isabela Rita de Carvalho Cunha ${ }^{4}$ \\ Fabio Luís Scarpa Bosso 5
}

\author{
${ }^{1}$ Hospital de Base do Distrito Federal ORCID 0000-0001-9357-5361 \\ ${ }^{2}$ Centro Universitário de Brasília ORCID 0000-0003-2265-0429 \\ ${ }^{3}$ Centro Universitário de Brasília ORCID 0000-0001-5374-6875 \\ ${ }^{4}$ Hospital Oftalmológico de Brasília ORCID 0000-0001-9322-844X \\ ${ }^{5}$ Hospital de Base do Distrito Federal ORCID 0000-0001-9142-0913 \\ E-mail pra correspondência: talita.trindadee94@gmail.com
}

\section{RESUMO}

O nervo troclear participa do movimento extrínseco dos olhos, por meio do músculo oblíquo superior. Seu acometimento não é frequente entre as lesões dos pares cranianos e é comumente congênito, relacionado à hipertensão ou ao trauma. A lesão causada por trauma cranioencefálico (TCE) geralmente ocorre em acidente automobilístico, com trauma orbitário direto ou frontal e tem maior incidência no gênero masculino. Os principais achados incluem hipertropia, diplopia vertical, limitação da depressão em adução e inclinação da cabeça para o lado oposto ao músculo oblíquo superior paralisado, manobra conhecida como sinal de Bielchowsky positivo. O objetivo deste estudo foi relatar o caso de um paciente de 54 anos, do gênero masculino, que apresentou diplopia após um TCE causado por um acidente automobilístico, tendo como principal hipótese, no momento da consulta, a paresia do IV nervo craniano à esquerda. A paresia dos nervos responsáveis pelos movimentos oculares após TCE é uma entidade com possibilidades de tratamento conservador e cirúrgico, que devem ser minuciosamente observados para melhor prognóstico e estratégia terapêutica.

Palavras-chave: paresia, nervo troclear, traumatismos craniocerebrais, oftalmologia.

\section{Paresis of the IV nerve with positive Bielschowsky sign: a case report}

\begin{abstract}
The trochlear nerve participates in the extrinsic movement of the eyes through the superior oblique muscle. Its involvement is not frequent among lesions of the cranial nerves and when affected the etiology may be congenital, traumatic or related to hypertension. Lesions caused by traumatic brain injury usually occur in vehicle collisions, related to direct or frontal orbital trauma, and are more frequent in men than women. The main signs include hypertropy, vertical diplopia,
\end{abstract}


limitation of adduction depression and inclination of the head to the opposite side of the paralyzed superior oblique muscle, a maneuver known as positive Bielchowsky sign. This study aims to report the case of a 54 years old male patient who presented diplopia after a traumatic brain injury caused by a car accident, which led to the main hypothesis of left cranial nerve palsy. The weakness of the nerves involved in the eye movement, after a traumatic brain injury, is elegible for conservative and surgical treatment, which should be throughly analised to provide the best prognosis and therapeutic strategy.

Keywords: paresis, trochlear nerve, craniocerebral trauma, ophthalmology.

\section{INTRODUÇÃO}

O nervo troclear participa do movimento extrínseco dos olhos, através do músculo oblíquo superior. Seu acometimento não é frequente entre as lesões dos pares cranianos ${ }^{1}$ e é comumente congênito, relacionado à hipertensão ou trauma ${ }^{2,3}$. Os principais achados incluem hipertropia, diplopia vertical, limitação da depressão em adução e inclinação da cabeça para o lado oposto ao músculo oblíquo superior paralisado, manobra conhecida como sinal de Bielchowsky positivo ${ }^{4}$.

\section{RELATO DE CASO}

Paciente A. R. da S., do sexo masculino, 54 anos, com história de acidente automobilístico culminando com trauma cranioencefálico há 9 anos. Evoluiu com diplopia sem melhora clínica. Ao exame físico, apresentava quadro motor típico de paresia unilateral de oblíquo superior à esquerda, evidenciado por sinal de Bielschowsky positivo, com a cabeça inclinada sobre o ombro direito. Havia hiperfunção de oblíquo inferior esquerdo 2+ quando realizado o Cover test e discreta anisometropia em "V". Em posição ortópica, o paciente apresentava hipertropia de 6 dioptrias prismáticas de olho esquerdo sobre o direito (E/D). Quando inclinado para a esquerda, hipertropia de 16 dioptrias de E/D e à inclinação para a direita, de 4 dioptrias de E/D. Apresentava melhor acuidade visual corrigida de 20/20 em ambos os olhos, biomicroscopia normal e sem quaisquer outras alterações ao exame oftalmológico, exceto por exciclotorsão à fundoscopia, bem evidenciada à retinografia (Figura 
1). Ao exame retinográfico do olho direito, foi demonstrada topografia normal (Figura 2). Foi realizada cirurgia segundo técnica de Apt e Call (1978), com recuo de $4 \mathrm{~mm}$ inferiormente e 0,4 mm lateralmente, resultando em retrocesso de $12 \mathrm{~mm}$ do músculo oblíquo inferior (OI). Após 3 meses, o paciente evoluiu com ortotropia ao exame físico e resolução completa da diplopia e da posição de cabeça, restando apenas leve desconforto à dextroversão extrema.

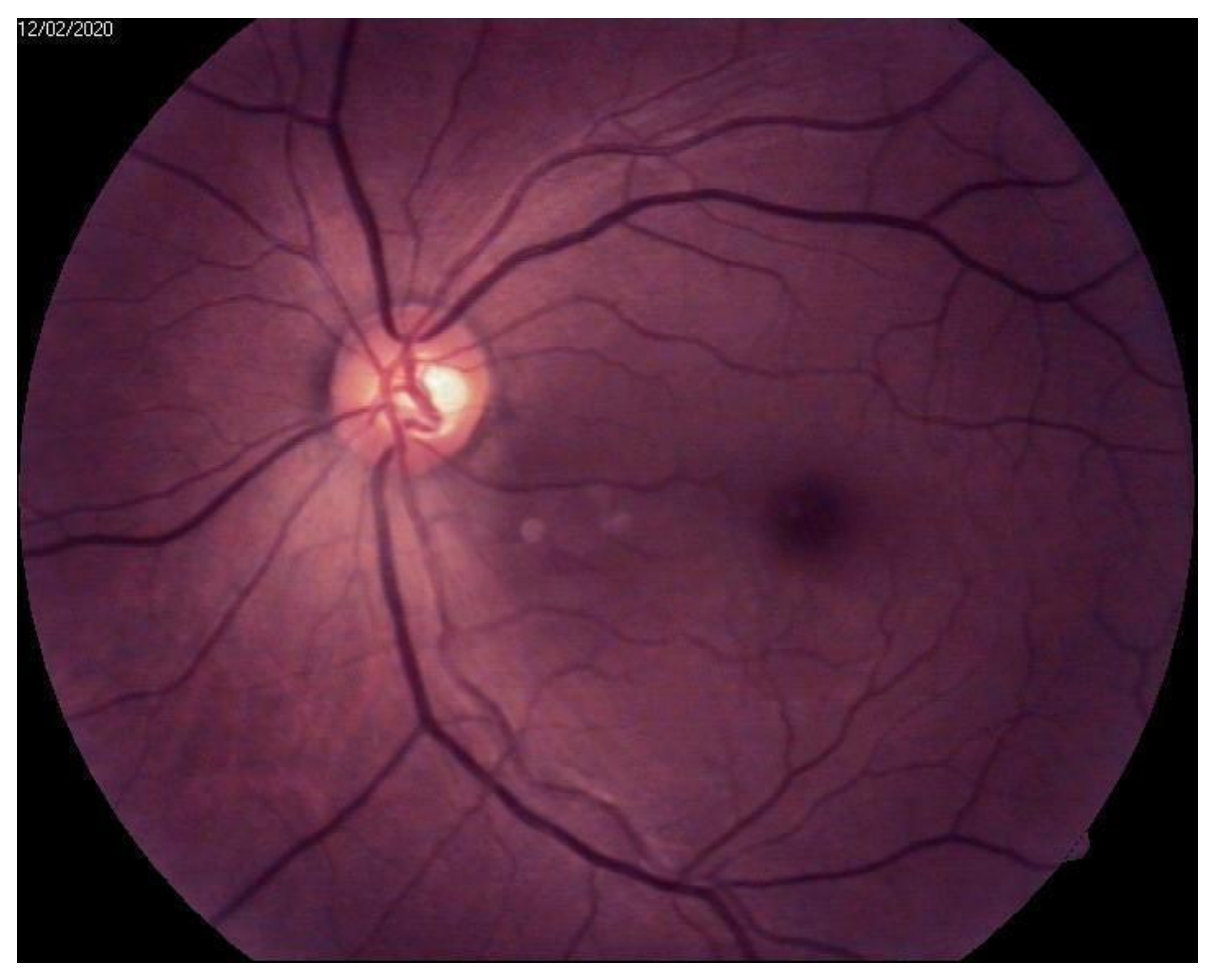

Figura 1: Exciclotorsão de olho esquerdo bem evidenciada à retinografia 


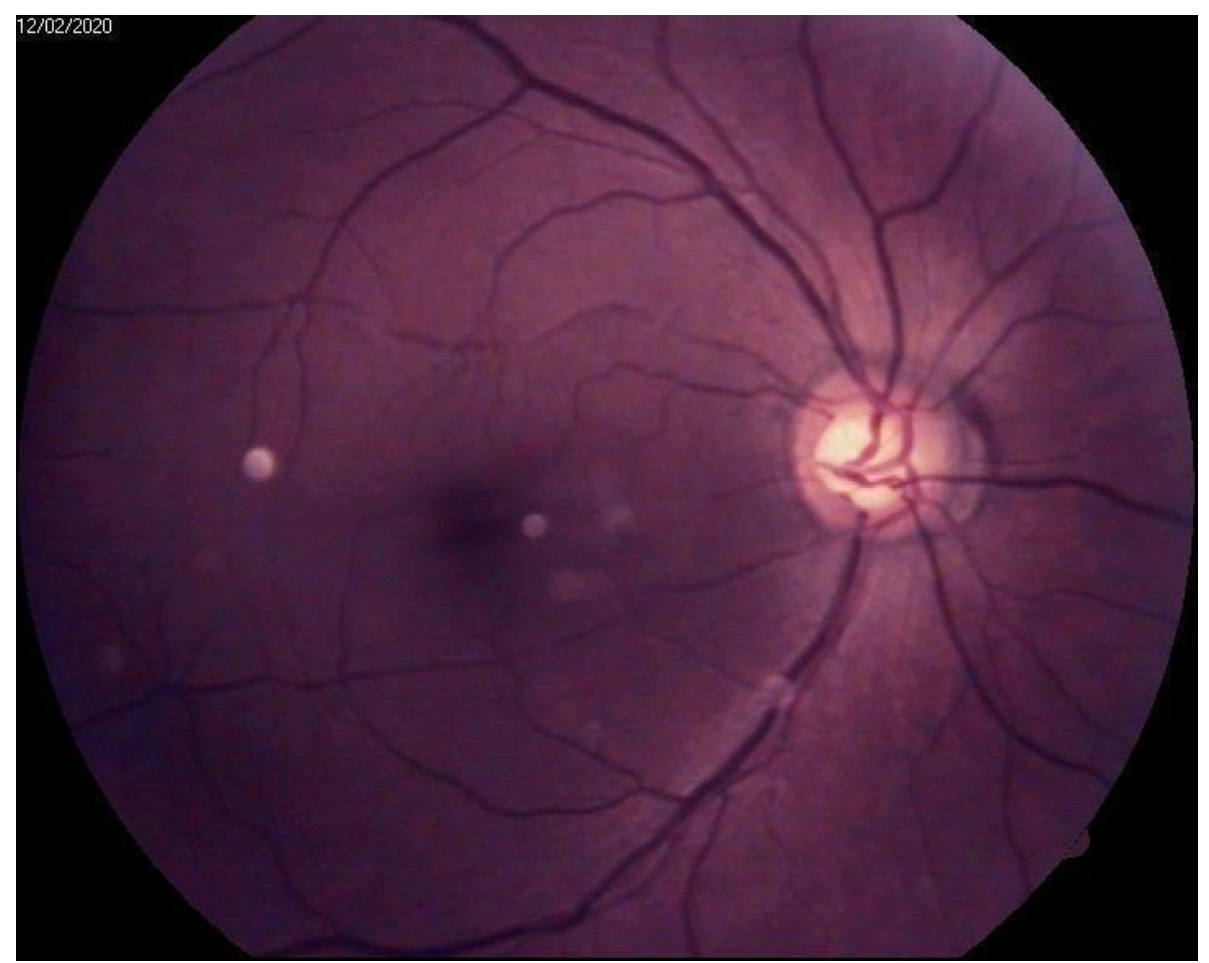

Figura 2: Retinografia de olho direito evidenciando fundo de olho com região macular em topografia normal.

\section{DISCUSSÃO}

O nervo troclear (IV par craniano), por ter o maior trajeto intracraniano, é o mais susceptível a paresias e a principal causa secundária são traumas de quaisquer magnitudes, de leves a graves. Em função desse trajeto após a saída do tronco cerebral, movimentos bruscos da cabeça e consequentemente do encéfalo podem causar seu estiramento e levar a paralisiado nervo ${ }^{5}$

A diplopia está entre os principais achados, relacionada a hipofunção do OS com consequente hiperfunção relativa do músculo OI. Em casos de hipofunção desse músculo causada por trauma, como no quadro do paciente apresentado, a diplopia é um sintoma frequente $^{6}$. Como tentativa de atenuar essa dupla visão, o paciente adota uma posição de inclinação da cabeça para o lado contrário do nervo lesado e o queixo para baixo ${ }^{7,8}$. Objetivase, assim, minimizar a hipertropia secundária à exciclotorsão do olho. Com isso, a inclinação 
da cabeça para frente reduz o desvio do eixo vertical, enquanto ao girar a face para o lado oposto do nervo comprometido, minimiza o desvio horizontal ${ }^{9,10}$.

A explicação para essa patogenia é relacionada ao cruzamento das fibras do nervo troclear no véu medular anterior, sendo o único nervo craniano que possui todas as suas fibras decussando após saírem do mesencéfalo. Seu local de decussação apresenta o nervo como uma estrutura fina, sendo facilmente traumatizada após a aplicação de forças externas, como em acidentes automobilísticos em que haja trauma orbitário direto ou frontal ${ }^{7}$.

A correção cirúrgica da paresia do oblíquo superior pode ser feita por fortalecimento desse músculo, pelo enfraquecimento do oblíquo inferior, pela recessão do músculo reto contralateral inferior ou reto ipsilateral superior. A técnica de escolha foi descrita por Apt e Call e propõe um recuo graduado dos músculos oblíquos inferiores utilizando como parâmetro o músculo reto inferior, que permite pequena variação na técnica para tratamento de hiperfunção moderada do OI, com utilização de recuos de $10 \mathrm{~mm} \mathrm{a} 12 \mathrm{~mm}^{12}$. Um prisma de ação vertical pode ser utilizado como alternativa no tratamento e, em casos de impossibilidade cirúrgica, a diplopia pode ser minimizada pela oclusão do olho afetado ${ }^{5}$. A escolha terapêutica objetiva um melhor resultado funcional e estético, além de favorecer a sociabilização do paciente.

\section{CONCLUSÕES}

A paresia do nervo troclear por causa traumática é de extrema importância na prática clínica, por ter bom prognóstico quando precocemente diagnosticada e tratada ${ }^{13,5}$. O principal achado é a diplopia, ocasionada pelo acometimento do músculo oblíquo superior ${ }^{6}$. Como forma de compensação e tentando minimizá-la, o paciente inclina a cabeça para frente e parao lado oposto do nervo comprometido, reduzindo o desvio do eixo vertical e horizontal, respectivamente ${ }^{9,10}$. Como tratamento, estão disponíveis correções cirúrgicas, sendo feitas por meio do fortalecimento do músculo, pelo enfraquecimento do oblíquo inferior ou ressecção 
do reto contralateral inferior ou reto ipsilateral superior ${ }^{11}$. A escolha terapêutica, baseada nas medidas e nos desbalanços secundários de músculos, mais comuns na forma primária, visa um melhor resultado estético e funcional com consequente melhora da qualidade de vida e sociabilização do paciente.

\section{REFERÊNCIAS}

1. Pereira, C. U., et al. Manifestações oculares de lesões na fossa craniana posterior. J Bras Neurocir 1996; 7(1): 33-44.

2. Maranhão-Filho, P., et al. Skew deviation e ocular tilt reaction versus paralisia do nervo troclear. Rev bras neurol 2015; 51(1): 0-0.

3. Dosunmu, E. O., et al. Incidence and etiology of presumed fourth cranial nerve palsy: a population-based study. Am J Ophthalmol 2018; 185: 110-114.

4. Tiffin, P. A. C., et al. Acquired palsy of the oculomotor, trochlear and abducens nerves. Eye 1996; 10(3): 377-384.

5. Dias, C. R. de S.; Goldchmit, M. Os Estrabismos. 1. ed. Rio de janeiro: Cultura Médica, 2011. V. 1000. 649 p.

6. Guichen Li, et al. Ocular movement nerve palsy after mild head trauma. World J Surg 2016; 94: 296-302.

7. Cannoni, L. F., et al. Lesões traumáticas de nervos cranianos. Arq bras neurocir 2012; 31(4): 184-194.

8. Park, U. C., et al. Clinical features and natural history of acquired third, fourth, and sixth cranial nerve palsy. Eye 2008; 22(5):691-696.

9. Moreira, A. T. R., et al. Paresia bilateral assimétrica de nervo troclear associada a trombose de seio transverso pós-otite-relato de caso. Arq Bras Oftalmol 2001; 64:153-5.

10. Diora, J. R., \& Plager, D. A. Sudden-onset trochlear nerve palsy: clinical characteristics and treatment implications. J AAPOS 2019; 23(6): 321-e1. 
11. Clifford, L., et al. Surgical management of superior oblique paresis using inferior oblique anterior transposition. J AAPOS 2015; 19(5): 406-409.

12. Apt, L., \& Call, N. B. Inferior oblique muscle recession. American journal of ophthalmology 1978; 85(1): 95-100.

13. Mollan, S. P., et al. Aetiology and outcomes of adult superior oblique palsies: a modern series. Eye 2009; 23(3): 640-644. 